\title{
Improving rectal dosimetry for patients with intermediate and high-risk prostate cancer undergoing combined high-dose-rate brachytherapy and external beam radiotherapy with hydrogel space
}

\author{
Michael Chao, FRANZCR',2,3, Darren Ow, MBBS², Huong Ho, BSc MSc', Yee Chan, FRACS 2,3, Daryl Lim Joon, FRANZCR², \\ Sandra Spencer, Dip App Sci Med Rad', Nathan Lawrentschuk, FRACS, PhD², Mario Guerrieri, FRANZCR', \\ Trung Pham, FRACS ${ }^{3}$, Kevin McMillan, FRACS ${ }^{3}$, Alwin Tan, FRACS ${ }^{4}$, Farshad Foroudi, FRANZCR, Doc Med Sci², \\ Johann Tang, FRANZCR ${ }^{5}$, Jason Wasiak, PhD², Madalena Liu, FRANZCR ${ }^{3}$, George Koufogiannis, FRACS ${ }^{3}$, \\ Chee Wee Cham, FRACS FRCS 4 , Damien Bolton, BA FRACS FRCS, MD2,3 \\ 'Genesis Cancer Care Victoria, Ringwood East, Australia, ${ }^{2}$ The Austin Hospital, Heidelberg, Australia, ${ }^{3}$ Ringwood Private Hospital, Ringwood \\ East, Australia, ${ }^{4}$ The Bays Hospital, Mornington, Australia, ${ }^{5}$ National University Hospital, Singapore
}

\begin{abstract}
Purpose: To report on rectal dosimetric and toxicity outcomes of intermediate and high-risk prostate cancer patients undergoing combined high-dose-rate (HDR) brachytherapy and external beam radiotherapy (EBRT) with or without hydrogel spacer (HS) insertion.

Material and methods: A total of 97 patients were analyzed in this study, with 32 patients (33\%) who had HS insertion compared with a preceding group of 65 patients $(67 \%)$ without HS. HS safety, the dosimetric effects on organs at risk (rectal, urethral, penile bulb, and bladder) as well as gastrointestinal (GI) and genitourinary toxicity were evaluated and compared between the two groups.

Results: The median prostate-rectal separation achieved with HS was $10 \mathrm{~mm}$ (range, 5-14 mm). There were no post-operative complications following HS insertion. Patients with HS had significantly lower radiation dose to the rectum across all rectal dose volumes from $\mathrm{rV}_{30}$ to $\mathrm{rV}_{80}$, whether in absolute volume (cc) or as percentage of contoured $\operatorname{OAR}(p<0.001)$. There was also significantly less acute $>$ grade 1 GI toxicity $(12.5 \%$ vs. $30.8 \%, p=0.05)$ and a trend towards less late grade 1 GI toxicity $(0 \%$ vs. $7.7 \% ; p=0.11)$ in the HS group compared to the non-HS group.

Conclusions: Insertion of HS in prostate cancer patients receiving combined HDR and EBRT is safe and has resulted in a significant radiation dose reduction to the rectum, resulting in significantly less acute GI toxicity and a trend towards less late GI toxicity.
\end{abstract}

J Contemp Brachytherapy 2019; 11, 1: 8-13 DOI: https://doi.org/10.5114/jcb.2019.82836

Key words: prostate cancer, hydrogel spacer, high-dose-rate, brachytherapy, rectal protection.

\section{Purpose}

The treatment options for men diagnosed with localized prostate cancer have continued to advance over time with relative efficacy seen with various regimes involving radical prostatectomy, external beam radiotherapy (EBRT), and/or brachytherapy [1,2]. Several reviews have also shown that the use of combination therapies such as low-dose-rate (LDR) or high-dose-rate (HDR) brachytherapy and EBRT have allowed for safe radiation dose escalation, which has translated into improved biochemical progression-free survival (bPFS) and metasta- sis-free survival (MFS) $[3,4,5]$. However, the benefits of radiation dose escalation with brachytherapy can come at a cost with increased genitourinary (GU) and gastrointestinal (GI) toxicity [6]. Despite advances in treatment delivery and target localization, the rectum remains a primary dose limiting normal tissue due to its close proximity to the prostate.

The use of hydrogel spacers (HS) between the prostate and rectum in recent years has gained considerable interest as it has been shown to safely and effectively increase perirectal spacing between the prostate and rectum, and thus reduce radiotherapy related GI toxicity $[7,8,9]$. In
Address for correspondence: Michael Chao, FRANZCR, Genesis Cancer Care Victoria, 36, Mt Dandenong Rd, Ringwood East, Victoria, Australia, phone: +61 03 88703300, fax: +61 03 88703388, e-mail: Michael.Chao@genesiscare.com
Received: 13.12 .2018

Accepted: 21.01.2019

Published: 28.02.2019 
this study, we evaluate the use of HS in 32 consecutive patients with intermediate- and high-risk prostate cancer treated with combination HDR brachytherapy and EBRT, and its impact on prostate and normal tissue dosimetry as well as GU and GI toxicity.

\section{Material and methods}

This retrospective study examined the clinical safety and efficacy of HS between the prostate and rectum (SpaceOAR ${ }^{\circledR}$, Augmenix Inc., Waltham, MA, USA) for men undergoing a course of combination HDR brachytherapy and EBRT at the Genesis Care Victoria. Institutional review board approval was obtained to conduct a retrospective review of our prospectively collected dataset of prostate cancer patients undergoing combination HDR brachytherapy and EBRT between 2010 and 2017. Ninety-seven men with intermediate- and high-risk prostate cancer were identified, of whom 32 consecutive patients also underwent HS insertion from 2014 as per our departmental protocol. Dosimetric parameters as well as cumulative acute and late toxicity for the 32 patients who underwent HS insertion were compared with the 65 consecutive preceding patients without HS insertion.

Our initial HDR brachytherapy was performed two weeks prior to EBRT. Patients were placed in the semi-lithotomy position under spinal anesthesia. Three gold fiducial markers were inserted into the prostate to facilitate image-guided radiation therapy (IGRT) for both HDR and EBRT. A total of 14-18 HDR catheters (OncoSmart ProGuide needles, Elekta, Stockholm, SW) were inserted into the prostate transperineally using a template technique ( $5 \mathrm{f}$ prostate stepper template, Elekta, Stockholm, SW) under transrectal ultrasound guidance with a Flex Focus 400 scanner (BK Medical Aps, Denmark). For patients treated from $2014(n=32)$, HS was injected into the perirectal fat to displace the anterior rectal wall posteriorly away from the prostate once all the HDR catheters were inserted. An $18 \mathrm{G}$ needle was inserted transperineally into the perirectal fat with the tip situated between the mid gland and apex. Hydrodissection with sterile saline was performed to open the potential space between Denonvilliers' fascia and the anterior rectal wall. Once this was confirmed, $10 \mathrm{ml}$ of HS was inserted [10].

Computed tomography (CT) simulation was performed using our Philips Brilliance Big Bore CT scanner (Philips Medical Systems, Fitchburg, WI) with $1 \mathrm{~mm}$ slice thickness. The dataset was then transferred electronically to our Oncentra ${ }^{\circledR}$ Brachytherapy Planning System v. 5.0 (Elekta, Stockholm, Sweden). The brachytherapy clinical tumor volume (CTV) was the prostate alone with a 2-3 mm margin to account for microscopic extension, except posteriorly where no margin was applied. The planning target volume (PTV) was the CTV. Dose goals were PTV: $\mathrm{V}_{100} \geq 90 \%, \mathrm{~V}_{150}<35 \%$, and $\mathrm{V}_{200}<15 \%$. The rectum was contoured as a whole solid structure beginning at $1.0 \mathrm{~cm}$ above the most superior level of the PTV to the anorectal junction. The urethra was contoured using the outer surface of the Foley catheter. The bladder and penile bulb were contoured as solid structures in its entirety (see Figure 1). Treatment plans were optimized using an anat- omy-based dwell time optimization approach (inverse planning simulated annealing method) with organs at risk (OARs) constraints based on RTOG 0321 (rectal $V_{75}$ $<1 \mathrm{cc}$, urethral $\mathrm{V}_{125}<1 \mathrm{cc}$, and bladder $\mathrm{V}_{75}<1 \mathrm{cc}$ ) [11] A total of 24 patients received an initial dose of $18 \mathrm{~Gy}$ in 3 fractions from 2010-2011, with the remaining 73 patients receiving 16 Gy in 2 fractions from 2012 onwards as per our departmental protocol. Treatment was delivered using a single implant by a remote afterloading Ir-192 source (Flexitron Afterloader v. 2.1.3, Elekta, Stockholm, Sweden), with a minimum of 6 hours between fractions.

EBRT was commenced within 2 weeks of HDR brachytherapy. A repeat CT simulation scan was performed post-HDR catheter removal with $3 \mathrm{~mm}$ slice thickness. The treatment plans were created on the Pinnacle v. 9.8 (Phillips Radiation Oncology Systems, Fitchburg, WI, USA) treatment planning system. The CTV was defined as the prostate and seminal vesicles. The CTV was expanded by $7 \mathrm{~mm}$ all around except posteriorly, where it was $5 \mathrm{~mm}$ to generate the PTV. All patients were treated with intensity modulated radiotherapy (IMRT) to a dose of 50.4 Gy in 1.8 Gy fractions on a Varian True Beam linear accelerator equipped with kilovoltage $(\mathrm{KV})$ cone beam $\mathrm{CT}$ (CBCT). All patients were treated with a full bladder and an empty rectum as per our departmental protocol.

All patients were evaluated at baseline, weekly during the EBRT, and every 3 months for the first year. They were subsequently followed every 6 months until their fifth year and yearly thereafter. Acute toxicity was defined as toxicity occurring during and within the first 90 days after completing their radiation treatment. Late toxicity was defined as toxicity occurring 90 days after their radiation treatment. The GI and GU toxicities were graded using

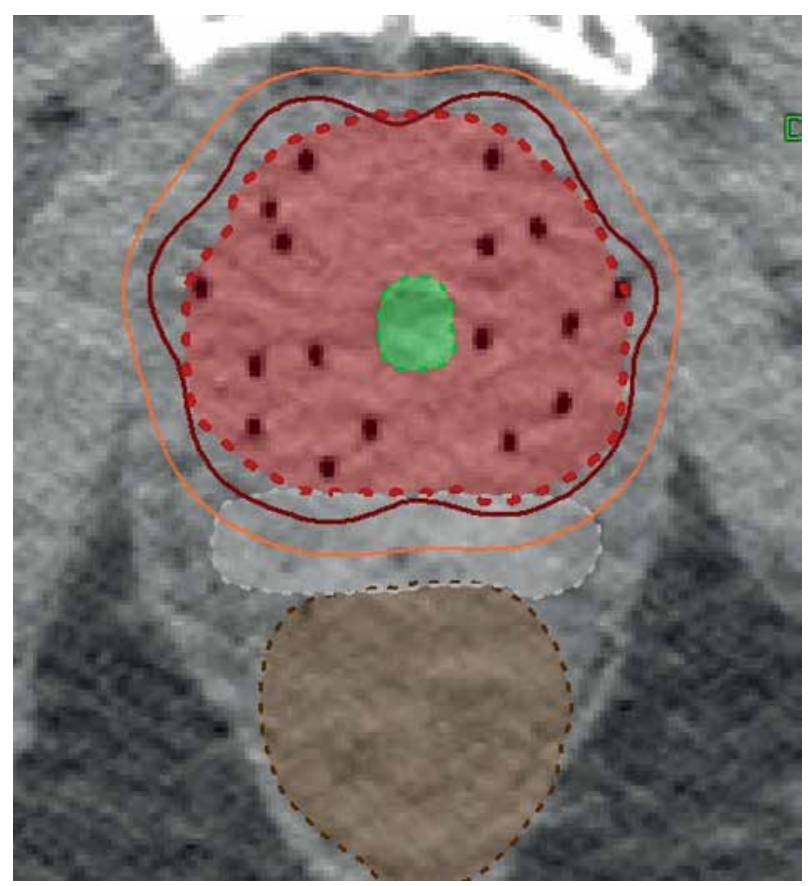

Fig. 1. CT HDR dosimetry with prostate PTV in light red, HS in white, urethra in green and rectum in brown. The 6 Gy and 8 Gy dose lines are outlined in orange and burgundy respectively 
the National Cancer Institute Common Terminology Criteria for Adverse Events v. 4.0 grading system [12].

\section{Statistical analysis}

Descriptive statistics were used to characterize some of the variables of interest. The Student's $t$-test was used to evaluate the level of significance of continuous variables with a normal distribution between the HS and non-HS plans. The Pearson's chi square test was used to evaluate the significance of categorical variables between the HS and non-HS plans. A $p$ value of $\leq 0.05$ was considered to be statistically significant. All analyses were performed using the IBM SPSS Statistics package (v. 24.0).

\section{Results}

The median follow-up for the entire cohort was 60 months (12-125 months). The median follow-up for the HS group was 42 months (12-63 months) compared to 65 months (26-125 months) for the non-HS group. The median age was 74 years (52-84 years). The median prostate specific antigen (PSA) was $9.7 \mathrm{ng} / \mathrm{ml}$. Forty-two patients had intermediate-risk disease, while the rest had high-risk disease. Androgen deprivation therapy (ADT) was delivered to the majority of patients $(89.7 \%)$. The ADT was recommended for six months in patients with intermediate-risk and for 24 months in high-risk disease. In patients who agreed to ADT, the treatment started at least three months before the commencement of radiotherapy. The patient characteristics are shown in Table 1.

HS was successfully implanted into all 32 patients who were planned to receive it. No post-operative complications such as rectal perforation or infection was recorded post HS insertion. The prostate to rectal separa- tion at mid gland was a median of $2 \mathrm{~mm}(0-5 \mathrm{~mm})$ in the non-HS group versus a median of $10 \mathrm{~mm}(5-14 \mathrm{~mm})$ in the HS group $(p<0.001)$.

The median prostate volume and HDR dose delivered to the target volume and OARs are shown in Table 2. There were no significant differences in median prostate volume, prostate $\mathrm{V}_{100}$, and prostate $\mathrm{V}_{200}$ between the two groups. The prostate $\mathrm{V}_{150}$ was significantly improved, with a lower value of $30.5 \%$ in the HS group compared with $33.5 \%$ in the non-HS group. The use of HS also resulted in significantly lower radiation dose to the rectum and urethra. The median rectal $\mathrm{V}_{75}$ (cc) was 0.45 (0-1.46) in the non-HS group compared to $0.0(0-0.22)$ for the HS group. The percentage relative reduction in dose was $100 \%$. All patients with $\mathrm{HS}$ met their rectal $\mathrm{V}_{75}$ constraint, whereas only $93.8 \%(61 / 65)$ in the non-HS group accomplished this restriction. The median urethral $\mathrm{V}_{125}$ (cc) was $0.06(0-1.01)$ in the non-HS group compared to 0.02 $(0-0.66)$ in the HS group. The percentage relative reduction in dose was $66.7 \%$. All patients with HS met their urethral $\mathrm{V}_{125}$ constraint, while 1 patient in the non-HS group failed to do so. There was no statistical difference in penile bulb radiation dose to $0.1,1$, or $2 \mathrm{cc}$ as percent of prescription dose. In addition, there was also no statistical difference in bladder $\mathrm{V}_{70}$ or $\mathrm{V}_{80}$ between the two groups.

The use of HS with HDR brachytherapy resulted in significantly lower radiation dose to the rectum across all rectal dose volumes from $\mathrm{rV}_{30}$ to $\mathrm{rV}_{80}$, whether in absolute volume (cc) or as percentage of contoured OAR (see Table 3). The impact of HS on rectal dose volumes was most substantial at the highest radiation doses with $\geq 95 \%$ relative reduction in rectal $\mathrm{V}_{60}$ to $\mathrm{V}_{80}$ whether in absolute volume (cc) or as percentage of contoured OAR. The impact of HS was still statistically significant at lower radiation

Table 1. Patients' characteristics of both with and without hydrogel spacer

\begin{tabular}{lccc} 
Characteristic & All patients & With HS $(n=32)$ & Without HS $(n=65)$ \\
\hline Median age (range) & $74.1(52.2-84.5)$ & $76.7(52.2-84.5)$ & $73.4(60.1-80.8)$ \\
\hline Median PSA $(\mathrm{ng} / \mathrm{ml})$ & $9.7(3.2-47)$ & $11.6(5.6-47)$ & $9.5(3.2-29.9)$ \\
\hline ADT & & & 55 \\
\hline Yes & 87 & 0 & 10 \\
\hline No & 10 & 0 & 5 \\
\hline Gleason score & 5 & 16 & 37 \\
\hline 6 & 53 & 3 & 13 \\
\hline 7 & 16 & 13 & 9 \\
\hline 8 & 22 & 0 & 1 \\
\hline 9 & 1 & 11 & 13 \\
\hline 10 & 18 & 16 & 37 \\
\hline Clinical stage & 48 & 5 & 15
\end{tabular}

HS - hydrogel spacer, PSA - prostate specific antigen, ADT - androgen deprivation therapy 
doses, but lower percentage relative reductions were seen in rectal dose volumes from $V_{30}$ to $V_{50}$.

The incidence of acute $\geq$ grade 1 GI toxicity in all patients was $24.7 \%$, with $1.1 \%$ developing grade 2 GI toxicity (see Table 4). No acute grade 3 GI toxicities were seen. The incidence of late $\geq$ grade 1 GI toxicity in all patients was $5.2 \%$. No late grade 2 or 3 GI toxicities were observed. There was significantly less acute $\geq$ grade 1 GI toxicity in the HS group compared to the non-HS group $(12.5 \%$ vs. $30.8 \% ; p=0.05)$. No patients in the HS group developed acute grade $2 \mathrm{GI}$ toxicity. There was also less late $\geq$ grade 1 GI toxicity in the HS group compared to the non-HS group, but this was not statistically significant $(0 \%$ vs. $7.7 \% ; p=0.11$ ). The incidence of acute $\geq$ grade $1 \mathrm{GU}$ toxicity in all patients was $91.6 \%$, with $1.1 \%$ developing grade 2 GU toxicity. No grade 3 acute toxicity was seen. The incidence of late $\geq$ grade $1, \geq 2$, and 3 GU toxicities in all patients were $44.3 \%, 6.2 \%$, and $4.1 \%$, respectively. There was no statistical difference in acute or late GU toxicity between the HS group compared to the non-HS group.

Table 2. Median HDR dose delivered to prostate and organs at risk

\begin{tabular}{|c|c|c|c|c|}
\hline Prescribed treatment dose (Gy) & All patients & With HS $(n=32)$ & Without HS $(n=65)$ & $p$-value \\
\hline Median prostate volume (cc) & $44.6(23.3-117.5)$ & $46.7(30.3-84.5)$ & $43.1(23.3-117.5)$ & 0.95 \\
\hline \multicolumn{5}{|l|}{ Prostate } \\
\hline$V_{100}$ & $95.4(84.3-98.9)$ & $94.9(84.3-97.4)$ & $95.6(88.1-98.9)$ & 0.05 \\
\hline $\mathrm{V}_{150}$ & $32.4(24.9-41.1)$ & $30.5(24.9-34.8)$ & $33.5(27-41.1)$ & 0.03 \\
\hline$V_{200}$ & $12.1(9.6-15.7)$ & $11.7(10.4-14.3)$ & $12.2(9.6-15.7)$ & 0.06 \\
\hline \multicolumn{5}{|l|}{ Rectal } \\
\hline $\mathrm{V}_{75}(\mathrm{cc})$ & $0.32(0-1.46)$ & $0(0-0.22)$ & $0.45(0-1.46)$ & $<0.001$ \\
\hline \multicolumn{5}{|l|}{ Urethral } \\
\hline$V_{125}(c c)$ & $0.04(0-1.01)$ & $0.02(0-0.66)$ & $0.06(0-1.46)$ & 0.02 \\
\hline \multicolumn{5}{|l|}{ Penile bulb } \\
\hline $0.1 \mathrm{cc}$ & $0.78(0.45-1.69)$ & $0.83(0.45-1.43)$ & $0.76(0.46-1.69)$ & 0.30 \\
\hline $1 \mathrm{cc}$ & $7.84(4.48-16.95)$ & $8.38(4.48-14.29)$ & $7.59(4.64-16.95)$ & 0.30 \\
\hline $2 \mathrm{cc}$ & $15.69(8.97-33.89)$ & $16.65(8.97-28.58)$ & $15.18(9.28-33.89)$ & 0.31 \\
\hline \multicolumn{5}{|l|}{ Bladder } \\
\hline $\mathrm{V}_{70}(\mathrm{cc})$ & $4.22(0.03-13.3)$ & $3.58(0.3-10.97)$ & $4.37(0.55-13.3)$ & 0.26 \\
\hline $\mathrm{V}_{80}(\mathrm{cc})$ & $2.60(0.00-9.5)$ & $2.30(0.00-7.6)$ & $2.86(0.14-9.5)$ & 0.25 \\
\hline
\end{tabular}

HS - hydrogel spacer

Table 3. Median radiation dose to rectum in patients with and without hydrogel spacer

\begin{tabular}{lccccccc} 
Rectal volume (in absolute) & $\mathrm{V}_{30}(\mathrm{cc})$ & $\mathrm{V}_{40}(\mathrm{cc})$ & $\mathrm{V}_{50}(\mathrm{cc})$ & $\mathrm{V}_{60}(\mathrm{cc})$ & $\mathrm{V}_{70}(\mathrm{cc})$ & $\mathrm{V}_{75}(\mathrm{cc})$ & $\mathrm{V}_{80}(\mathrm{cc})$ \\
\hline In absolute volume $(\mathrm{cc})$ & & & & & & \\
\hline All & $14.40(4.4-40)$ & $7.40(0.9-21.8)$ & $3.70(0.07-11.8)$ & $1.70(0-6.1)$ & $0.65(0-11.1)$ & $0.32(0-1.46)$ & $0.10(0-0.56)$ \\
\hline - Hydrogel spacer & $16.50(6.6-40)$ & $8.60(3.2-21.8)$ & $4.30(1.4-11.8)$ & $2.10(0.31-0.6)$ & $0.86(0-11.1)$ & $0.45(0-1.46)$ & $0.20(0-0.56)$ \\
\hline+ Hydrogel spacer & $10.50(4.4-22.3)$ & $3.60(0.9-9.9)$ & $1.00(0.07-4.2)$ & $0.10(0-1.6)$ & $0.00(0-0.45)$ & $0.00(0-0.22)$ & $0.00(0-0.08)$ \\
\hline \% relative reduction & $36.4 \%$ & $58.1 \%$ & $76.7 \%$ & $95.2 \%$ & $100 \%$ & $100 \%$ & $100 \%$ \\
\hline$p$-value & $<0.001$ & $<0.001$ & $<0.001$ & $<0.001$ & $<0.001$ & $<0.001$ & $<0.001$ \\
\hline In percentage (\%) & $\mathrm{V}_{30} \%$ & $\mathrm{~V}_{40} \%$ & $\mathrm{~V}_{50} \%$ & $\mathrm{~V}_{60} \%$ & $\mathrm{~V}_{70} \%$ & $\mathrm{~V}_{75} \%$ & $\mathrm{~V}_{80} \%$ \\
\hline All & $18.50(3.9-44.9)$ & $9.30(0.8-24.3)$ & $4.50(0.07-13.1)$ & $2.00(0-6.5)$ & $0.74(0-2.5)$ & $0.41(0-1.4)$ & $0.12(0-0.66)$ \\
\hline- Hydrogel spacer & $20.60(10.7-44.9)$ & $10.70(3.2-21.8)$ & $5.50(2.1-13.3)$ & $2.70(0.5-6.5)$ & $1.10(0.01-2.5)$ & $0.55(0-1.4)$ & $0.21(0-0.66)$ \\
\hline+ Hydrogel spacer & $12.20(3.9-26.4)$ & $4.60(0.8-17.7)$ & $1.40(0.07-4.2)$ & $0.10(0-2)$ & $0.00(0-0.6)$ & $0.00(0-0.25)$ & $0.00(0-0.09)$ \\
\hline$\%$ relative reduction & $40.8 \%$ & $57 \%$ & $74.5 \%$ & $96.3 \%$ & $100 \%$ & $100 \%$ & $100 \%$ \\
\hline$p$-value & $<0.001$ & $<0.001$ & $<0.001$ & $<0.001$ & $<0.001$ & $<0.001$ & $<0.001$
\end{tabular}


Table 4. Impact of hydrogel spacer on acute and late gastrointestinal/genitourinary toxicity

\begin{tabular}{|c|c|c|c|c|c|}
\hline Toxicity & Toxicity grade & All & No HS & HS & $p$-value \\
\hline \multicolumn{6}{|c|}{ Genitourinary } \\
\hline \multirow[t]{2}{*}{ Acute } & $\geq$ Grade 1 & $91.6 \%$ & $92.3 \%$ & $83.3 \%$ & 0.22 \\
\hline & Grade 2 & $1.1 \%$ & $1.5 \%$ & $0 \%$ & 0.48 \\
\hline \multirow[t]{3}{*}{ Late } & $\geq$ Grade 1 & $44 \%$ & $43.1 \%$ & $46.7 \%$ & 0.74 \\
\hline & $\geq$ Grade 2 & $6.3 \%$ & $7.7 \%$ & $3.3 \%$ & 0.40 \\
\hline & Grade 3 & $4.2 \%$ & $6.2 \%$ & $3.3 \%$ & 0.57 \\
\hline \multicolumn{6}{|c|}{ Gastrointestinal } \\
\hline \multirow[t]{2}{*}{ Acute } & $\geq$ Grade 1 & $25.3 \%$ & $30.8 \%$ & $13.3 \%$ & 0.05 \\
\hline & Grade 2 & $1.1 \%$ & $1.5 \%$ & $0 \%$ & 0.48 \\
\hline Late & Grade 1 & $5.3 \%$ & $7.7 \%$ & $0 \%$ & 0.11 \\
\hline
\end{tabular}

HS - hydrogel spacer

\section{Discussion}

This is the largest Australian study reporting on the use of HS in prostate cancer patients undergoing HDR brachytherapy with EBRT. The use of HS in our study was found to be safe and efficacious. All 32 patients planned for HS insertion were successfully implanted. No significant adverse events were reported in our study. This is consistent with the reports from Strom et al. and Yeh et al. where a combined 426 patients were successfully implanted without complications $[13,14]$. However, a recent study of 18 patients from $\mathrm{Wu}$ et al. reported a perineal abscess that developed 1 month following HS insertion and HDR implant, requiring antibiotics and drainage [15]. HS has also been inserted successfully in a patient undergoing salvage HDR brachytherapy after initial brachytherapy [16].

The use of HS resulted in a mean prostate rectal separation of $10 \mathrm{~mm}$. This is consistent with a separation distance of $12 \mathrm{~mm}$ reported by Strom et al., but less than that reported by Yeh et al. of $16 \mathrm{~mm}$ in HDR studies examining the use of HS $[13,14]$. Other EBRT specific studies, such as Mariados et al., Prada et al., and Pinkawa et al. were able to achieve prostate rectal separation distances between $12-15 \mathrm{~mm}[10,17,18]$. In our study, despite a smaller mean separation distance of $10 \mathrm{~mm}$, we were still able to significantly influence rectal dose endpoints with rectal $V_{75}$ reduced by $100 \%$.

The use of HS significantly reduced rectal irradiation from $V_{30}$ to $V_{80}$ compared to the non-HS group. This is important because rectal toxicity is correlated with the volume of rectum receiving a particular threshold dose of radiation. The study by $\mathrm{Wu}$ et al. reported significant relative reductions in rectal $V_{50}$ to $V_{80}$ whether in absolute volume (cc) or as percentage of contoured OAR [15]. The median rectal $\mathrm{V}_{75}$ (cc) was 0.12 in the non-HS group compared to $<0.005$ in the HS group. The percentage relative reduction was almost $100 \%$. This is consistent with our study, where the median rectal $V_{75}$ (cc) was $0.45(0-1.46)$ in the non-HS group compared to $0.00(0-0.22)$ for the HS group. In addition, the percentage relative reduction in dose was also $100 \%$. Of note, all patients with HS met their rectal $\mathrm{V}_{75}$ constraint, whereas only $93.8 \%(61 / 65)$ in the non-HS group accomplished this. For patients who fail to meet their rectal dose constraint, we can either compromise PTV coverage by accepting a lower prostate $\mathrm{V}_{100}$ or accept potentially higher rectal toxicity. Yeh et al. also reported lower mean and maximum doses to the rectum with the use of HS [14]. The average mean dose to the rectum was $36 \%$ of prescribed dose with no-HS cohort, decreasing to $29 \%$ with HS cohort. In addition, the average maximum dose to the rectum was $95 \%$ of prescribed dose without HS, decreasing to $78 \%$ with HS. Strom et al. reported a significantly reduced rectal D2 cc of $60 \%$ without HS compared to $47 \%$ with HS [13].

The use of combined brachytherapy (HDR or LDR) with EBRT can lead to increased GI and GU toxicity [11]. When HDR is combined with EBRT, the risk of late grade $3 \mathrm{GI}$ toxicity can be as high as $7 \%$. In the two phase 3 randomized studies comparing HDR with EBRT versus EBRT alone, the risk of late grade 3 GI toxicity was $3.9 \%$ in the Sathya et al. study and $7 \%$ in the Hoskin et al. study $[19,20]$. A phase 2 RTOG 0321 study reported a $2.6 \%$ combined late grade 3 GI/GU toxicity [11]. A retrospective study by Spratt et al. reported late grade 2 and 3 GI toxicity of $4.1 \%$ and $1.4 \%$, respectively for combined brachytherapy and EBRT after a median follow-up of 5.3 years [21]. Yeh et al. delivered aggressive dose escalated HDR brachytherapy of 16 Gy in 2 fractions combined with EBRT of 59.4 Gy in 33 fractions in 326 patients [14]. After a median follow-up of 16 months, the reported rates of acute rectal grade 1 and 2 toxicities were $37.4 \%$ and $2.8 \%$, respectively. No acute rectal grade 3 or 4 toxicities were reported. The rates of late rectal grade 1 and 2 toxicities were $12.7 \%$ and $1.4 \%$, respectively. In addition, two patients $(0.7 \%)$ developed late grade 3 rectal toxicity. In our study, the risk of acute $\geq$ grade 1 GI toxicity was significantly lower in the HS group at $12.5 \%$ compared with $30.8 \%$ in the non-HS group. The risk of acute grade 2 GI toxicity was minimal in both groups. However, the risk of late grade 1 toxicity 
was $0 \%$ in the HS group compared to $7.7 \%$ in the nonHS group. Although, this was not found to be statistically significant, the absolute difference between the two groups approaches that seen in the randomized Mariados et al. study of HS in prostate EBRT [7]. Although the risk of both acute and late rectal toxicity is higher in the study by Yeh et al., this is a consequence of their aggressive dose escalation [14]. Despite an EBRT dose of 59.4 Gy following HDR brachytherapy, the reported late rectal grade 3 toxicity of $0.7 \%$ can be considered low compared to the two randomized studies. The use of HS may well have contributed to the lower than expected late rectal grade 3 toxicity.

Although we saw a significant difference in urethral $\mathrm{V}_{125}(\mathrm{cc})$ between the two groups, the absolute difference was small (0.02 cc in the HS group compared with 0.06 $\mathrm{cc}$ in the non-HS group). In addition, the urethral $\mathrm{V}_{125}$ was well within the recommended constraint of $<1 \mathrm{cc}$ for the majority of patients. As such, we saw no difference in acute or late GU toxicity between the two groups. It is unlikely that the use of HS would have accounted for the difference in urethral $V_{125}$. Rather, it is likely attributable to the learning curve inherent in the adoption of any new techniques and the improvement in dosimetry seen with the latter cohort of HS patients.

This study also had a number of limitations. A retrospective case series methodology was used, which may have introduced a potential patient selection bias. However, the median prostate volumes and median dose delivered (prostate $\mathrm{V}_{100}$ ) were not significantly different, inferring any improvements seen in OAR dosimetry was the effect of the HS. Secondly, our median follow-up for the HS group was shorter than that for the non-HS group. This may potentially lead to the underestimation of late GI toxicity in the HS group.

\section{Conclusions}

The use of HS is safe and effective for patients treated with combined HDR brachytherapy and EBRT. The insertion of HS was successful in all patients who were planned to receive it. This created on average a rectal prostatic separation of $10 \mathrm{~mm}$, which resulted in marked improvements in rectal OAR dosimetry leading to a trend towards fewer acute and late GI toxicity.

\section{Disclosure}

Authors report no conflict of interest.

\section{References}

1. Hannoun-Lévi JM. Brachytherapy for prostate cancer: Present and future. Cancer Radiother 2017; 21: 469-472.

2. Stish BJ, Davis BJ, Mynderse LA et al. Brachytherapy in the management of prostate cancer. Surg Oncol Clin N Am 2017; 26: 491-513.

3. Skowronek J. Current status of brachytherapy in cancer treatment - short overview. J Contemp Brachytherapy 2017; 9: 581-589.

4. Skowronek J. Low-dose-rate or high-dose-rate brachytherapy in treatment of prostate cancer - between options. J Contemp Brachytherapy 2013; 5: 33-41.
5. Morton GC. High-dose-rate brachytherapy boost for prostate cancer: rationale and technique. J Contemp Brachytherapy 2014; 6: 323-330.

6. Kee DLC, Gal J, Falk AT et al. Brachytherapy versus external beam radiotherapy boost for prostate cancer: Systematic review with meta-analysis of randomized trials. Cancer Treat Rev 2018; 70: 265-271.

7. Mariados N, Sylvester J, Shah D et al. Hydrogel spacer prospective multicenter randomized controlled pivotal trial: dosimetric and clinical effects of perirectal spacer application in men undergoing prostate image guided intensity modulated radiation therapy. Int J Radiat Oncol Biol Phys 2015; 92: 971-977.

8. Pinkawa M, Berneking V, Schlenter M et al. Quality of life after radiation therapy for prostate cancer with a hydrogel spacer: 5-year results. Int J Radiat Oncol Biol Phys 2017; 99: 374-377.

9. Chao M, Ho H, Chan Y et al. Prospective analysis of hydrogel spacer for patients with prostate cancer undergoing radiotherapy. BJU Int 2018; 122: 427-433.

10. Pinkawa M, Corral NE, Caffaro M et al. Application of a spacer gel to optimize three-dimensional conformal and intensity modulated radiotherapy for prostate cancer. Radiother Oncol 2011; 100: 436-441.

11. Hsu IC, Bae K, Shinohara K et al. Phase II trial of combined high-dose-rate brachytherapy and external beam radiotherapy for adenocarcinoma of the prostate: preliminary results of RTOG 0321. Int J Radiat Oncol Biol Phys 2010; 78: 751-758.

12. https://www.eortc.be/services/doc/ctc/CTCAE_4.03 _2010-06-14_QuickReference_5x7.pdf

13. Strom TJ, Wilder RB, Fernandez DC et al. A dosimetric study of polyethylene glycol hydrogel in 200 prostate cancer patients treated with high-dose rate brachytherapy +/-intensity modulated radiation therapy. Radiother Oncol 2014; 111: 126-131.

14. Yeh J, Lehrich B, Tran C et al. Polyethylene glycol hydrogel rectal spacer implantation in patients with prostate cancer undergoing combination high-dose-rate brachytherapy and external beam radiotherapy. Brachytherapy 2016; 15: 283-287.

15. Wu SY, Boreta $\mathrm{L}, \mathrm{Wu} \mathrm{A}$ et al. Improved rectal dosimetry with the use of SpaceOAR during high-dose-rate brachytherapy. Brachytherapy 2018; 17: 259-264.

16. Hepp R, Eggert T, Schabl G et al. Salvage high-doserate brachytherapy for prostate cancer persistence after brachytherapy: repeated use of a polyethylene glycol hydrogel spacer. J Contemp Brachytherapy 2018; 10: 169-173.

17. Hamstra DA, Mariados N, Sylvester J et al. Continued benefit to rectal separation for prostate radiation therapy: final results of a phase iii trial. Int J Radiat Oncol Biol Phys 2017; 97: 976-985.

18. Prada PJ, Fernandez J, Martinez AA et al. Transperineal injection of hyaluronic acid in anterior perirectal fat to decrease rectal toxicity from radiation delivered with intensity modulated brachytherapy or EBRT for prostate cancer patients. Int J Radiat Oncol Biol Phys 2007; 69: 95-102.

19. Sathya JR, Davis IR, Julian JA et al. Randomized trial comparing iridium implant plus external-beam radiation therapy with external-beam radiation therapy alone in node-negative locally advanced cancer of the prostate. J Clin Oncol 2005; 23: 1192-1199.

20. Hoskin PJ, Rojas AM, Bownes PJ et al. Randomised trial of external beam radiotherapy alone or combined with highdose-rate brachytherapy boost for localised prostate cancer. Radiother Oncol 2012; 103: 217-222.

21. Spratt DE, Zumsteg ZS, Ghadjar P et al. Comparison of high-dose (86.4 Gy) IMRT vs combined brachytherapy plus IMRT for intermediate-risk prostate cancer. BJU Int 2014; 114: 360-367. 